\title{
Cost sharing of cooperating queues in a Jackson network *
}

\author{
Judith Timmer ${ }^{\dagger} \quad$ Werner Scheinhardt ${ }^{\dagger}$
}

November 24, 2011

\begin{abstract}
We consider networks of queues in which the independent operators of individual queues may cooperate to reduce the amount of waiting. More specifically, we focus on Jackson networks in which the total capacity of the servers can be redistributed over all queues in any desired way. If we associate a cost to waiting that is linear in the queue lengths, it is known how the operators should share the available service capacity to minimize the long run total cost.

We answer the question whether or not (the operators of) the individual queues will indeed cooperate in this way, and if so, how they will share the cost in the new situation. One of the results is an explicit cost allocation that is beneficial for all operators. The approach used also works for other cost functions, such as the server utilization.
\end{abstract}

Keywords: Jackson network, cooperation, cost allocation, game theory, capacity allocation.

\section{Introduction}

Consider a queueing network consisting of different queues, and assume that each of these is operated by a different, independent operator. By working together (in some way), the operators can optimize the performance of the network (in some sense), leading to a social optimum with minimum total cost for the operators. On the other hand, individual operators will try to minimize their own cost, and will only cooperate if this is to their own benefit. This explains our idea of analyzing such networks using cooperative game theory.

In particular, we can view the independent operators as decision makers (or players) in a so-called cooperative cost game, see e.g. [10]. In such a game, the players make binding agreements (as opposed to noncooperative games) to jointly optimize the total cost they need to pay, and then try to share this cost by finding a fair cost allocation. Typically, a cost allocation is fair if it lowers the cost for each possible coalition (i.e. for any group of players). If this is not the case, then full cooperation is not beneficial, but there may still be partial cooperation between some (but not all) players.

When we try to model a queueing network as described above, there are a number of choices to be made. First of all, $(i)$ one can think of a variety of ways in which the operators may work together, including sharing service capacity, sharing buffer capacity, or changing the routing structure; moreover any of these can be done dynamically or statically (i.e. dependent

\footnotetext{
${ }^{*}$ In the Netherlands, the 3 universities of technology have formed the 3TU.Federation. This article is the result of joint research in the 3TU.Centre of Competence NIRICT (Netherlands Institute for Research on ICT).

${ }^{\dagger}$ Department of Applied Mathematics, University of Twente, P.O. Box 217, 7500 AE Enschede, The Netherlands. E-mail: \{j.b.timmer, w.r.w.scheinhardt\}@utwente.nl
} 
on the current state of the network or not). Furthermore, (ii) different network topologies may be considered. Similarly, (iii) traffic characteristics, i.e. the behaviour of the arrival process(es) and service demands, can be modeled in many ways. Finally, $(i v)$ the performance of the total network and its individual queues, and the associated cost, can be measured in many ways.

In this paper we study an initial model to investigate whether this line of research is useful to pursue. In this model we make the following assumptions: $(i)$ the different servers are able to share their service capacity, and do so in a static way; (ii, iii) the network is an $n$-node Jackson network, see e.g. [13]: customers arrive to (some of the) nodes according to independent Poisson processes, then (after service) move to another queue, according to some routing probabilities, etcetera, until they leave the system; service times are all exponentially distibuted; $(i v)$ we take the cost at queue $i$ to be proportional to the long run expected queue length, or equivalently (by Little's law), proportional to the expected sojourn time of customers in queue $i$; furthermore, the total cost of a group of queues is just the sum of the costs of the individual queues (thus, there is no cost associated to the cooperation itself).

In earlier work [12] we treated a special case of our Jackson model, in which the network is a traditional tandem queue. We found that even this very elementary model exhibits some interesting and non-trivial behavior. In the current paper we treat the more general model, but also find better results. In particular we present a cost allocation which is shown to be beneficial for all operators.

In the queueing literature there are many references in which the queues in a network cooperate (sharing capacity, pooling resources, etc.) to reach some form of optimality, e.g. $[2,9]$. However, in most cases the whole network is (implicitly) supposed to be run by a single operator. The combination of the queueing model with game theory, in which independent operators are only willing to cooperate if a good cost allocation can be found while they remain independent, is to the best of our knowledge hardly studied so far. Some related references are the following. González and Herrero [7] study several medical departments that may share an operating theatre. The cost of each department is linear in the capacity needed to satisfy a maximum on the expected waiting time of its patients. It is shown that cooperation reduces the total cost, and that a cost allocation can be determined based on the Shapley value. In [4], García-Sanz et al. extend this model and study cooperation among Markovian queues that share a common server with preemptive priority discipline. The authors show that a cost allocation proportional to the arrival rates is fair. More recently, Anily and Haviv [3] study cooperation in service capacity management. A number of servers pool their capacities and customers. The cost of cooperation is the mean number of customers in the pooled system. It is shown that fair cost allocations always exist. In particular, servers with large capacities may receive payment for cooperation. In these three papers, the servers cooperate by means of pooling. One way in which our paper contributes is that we consider cooperation in a network of queues, while preserving the autonomy of the individual queues; we do not allow for pooling.

In [5] cooperative game theory is used to study resource allocation in dynamic ad-hoc networks, assuming that the cost function is superadditive. We also like to mention [6] where a cooperative game is considered in which countries can form coalitions to optimize the routing of (international) teletraffic streams. For a trial data set they identify the most important members of the possible coalitions and the way in which benefits could be shared. Finally, we mention Altman et al. [1] who give an extensive survey on networking games. The models and papers discussed in this reference mostly deal with non-cooperative game theory, the only 
exception being a short section focused on bargaining games. This strengthens our belief that the problem formulation and approach in the current paper have not been studied before.

We end this section with a short overview of the remainder of the paper: in Section 2 we introduce the model in more detail with the optimal capacity allocation, and recall the basics of cooperative game theory. Based on this we introduce the so-called Jackson games. Then, in Section 3 we first derive results for two- and three-node Jackson games, followed by an explicit cost allocation in Theorem 8, showing that the core is never empty. In Section 4 we focus on a special case, viz. the tandem games as earlier presented in [12], and on an extension in which we choose the server utilization, rather than the expected queue length, as performance measure. We conclude in Section 5, also sketching some main lines for future research.

\section{$2 \quad$ Model and preliminaries}

In this section we first introduce our model in detail, and derive the optimal capacity allocation. Then we recall the basics of cooperative game theory, focusing on the solution concept of the core, which is the set of all cost allocations that are acceptable to all possible coalitions of queues. The last subsection shows how our Jackson model fits into the framework of cooperative game theory.

\section{$2.1 \quad$ Model}

We consider an $n$-node Jackson network, denoting the set of all queues by $N=\{1,2, \ldots, n\}$. External customers arrive at queue $i$ according to a Poisson process with rate $\lambda_{i}^{0}$. After finishing its service at queue $i$, any customer joins queue $j$ with probability $p_{i j}$ independent of all else, and leaves the network with probability $1-\sum_{j \in N} p_{i j}$. We are only concerned with the local arrival rates $\lambda_{i}$ to queue $i$, which follow from the traffic equations $\lambda_{j}=\lambda_{j}^{0}+\sum_{i \in N} p_{i j} \lambda_{i}$ for all $j \in N$. The exponential service capacity at queue $i$ is given by $\mu_{i}$, where we assume $\mu_{i}>\lambda_{i}$. The cost incurred at queue $i$ is represented by the long run expected queue length $\lambda_{i} /\left(\mu_{i}-\lambda_{i}\right)$. Furthermore, the total cost of any subset $S \subseteq N$ of queues is the sum of the costs of the individual queues in $S$.

Importantly, we assume that the queues in any subset $S$ may cooperate to improve their performance and save on costs. Cooperation here means that the queues in $S$ may redistribute their service capacities among each other. Denoting the service capacity of queue $i$ after redistribution by $m_{i}$, this leads to the following optimization problem for the set $S$ :

$$
\begin{aligned}
\min _{m_{i}, i \in S} & \sum_{i \in S} \frac{\lambda_{i}}{m_{i}-\lambda_{i}} \\
\text { s.t. } & \sum_{i \in S} m_{i}=\sum_{i \in S} \mu_{i} .
\end{aligned}
$$

To solve this problem, rewrite it as

$$
\min _{\alpha, m_{i}, i \in S} \sum_{i \in S} \frac{\lambda_{i}}{m_{i}-\lambda_{i}}-\alpha\left(\sum_{i \in S} m_{i}-\sum_{i \in S} \mu_{i}\right),
$$


where $\alpha$ is the Lagrange multiplier. Taking derivatives leads to the solution [8, p. 63]

$$
m_{i, S}=\lambda_{i}+\frac{\sqrt{\lambda_{i}}}{\sum_{k \in S} \sqrt{\lambda_{k}}} \sum_{k \in S}\left(\mu_{k}-\lambda_{k}\right) \quad i \in S
$$

which is denoted by $m_{i, S}$ to stress the dependence on the set $S$. In this solution, the total excess capacity $\sum_{k \in S}\left(\mu_{k}-\lambda_{k}\right)$ is distributed proportional to the square root of the demand rate. The corresponding minimal cost for the set $S$ of (cooperating) queues is

$$
c(S)=\frac{\left(\sum_{k \in S} \sqrt{\lambda_{k}}\right)^{2}}{\sum_{k \in S}\left(\mu_{k}-\lambda_{k}\right)}
$$

Notice that queue $i$ contributes the amount $\sqrt{\lambda_{i}} \sum_{k \in S} \sqrt{\lambda_{k}} / \sum_{k \in S}\left(\mu_{k}-\lambda_{k}\right)$ to the cost for $S$.

\subsection{Preliminaries on cooperative cost games}

A cooperative cost game is represented by a pair $(N, c)$. The set $N=\{1, \ldots, n\}$ is the set of players. A coalition $S$ is a (nonempty) group of players, that is, a nonempty subset of $N$. The cost function $c$ assigns to each coalition $S$ a certain cost $c(S)$.

In our analysis we will need the concept of marginal vectors, and monotonicity. Let $\sigma=\sigma(1) \ldots \sigma(n)$ be a permutation of the player set, where $\sigma(k)$ is the player in position $k$. Denote by $P_{\sigma}(i)=\left\{j \in N \mid \sigma^{-1}(j)<\sigma^{-1}(i)\right\}$ the set of players in positions before player $i$. Now imagine that the players enter a room one by one in the ordering indicated by $\sigma$, and that each player has to pay the marginal contribution to the total cost when he enters the room. Then player $i$ pays

$$
m_{i}^{\sigma}=c\left(P_{\sigma}(i) \cup\{i\}\right)-c\left(P_{\sigma}(i)\right) .
$$

The vector $m^{\sigma}=\left(m_{1}^{\sigma}, \ldots, m_{n}^{\sigma}\right)$ is called the marginal vector corresponding to the permutation $\sigma$. Further, a cost game is called monotone increasing (respectively decreasing) if $S \subseteq T$ implies $c(S) \leq c(T)$ (respectively $c(S) \geq c(T)$ ).

A game is additive if the coalitional costs are additive, $c(S)=\sum_{k \in S} c(\{k\})$. If for any two disjoint coalitions $S$ and $T$ of players it is beneficial to cooperate, we say the game is subadditive. In this case, cooperation never leads to higher cost when compared to working separately:

$$
c(S \cup T) \leq c(S)+c(T) .
$$

Remark 1. Notice that in a subadditive game the choice $T=N \backslash S$ in (4) implies $c(N) \leq$ $c(S)+c(N \backslash S)$. Thus, if we split the coalition $N$ of all players in two parts, namely the coalitions $S$ and $N \backslash S$, then the total cost does not decrease. This is an incentive for all the players in coalition $N$ to cooperate.

The main question that remains is how the total joint cost $c(N)$ should be allocated among the players. A first step towards selecting a good and fair cost allocation is to consider allocations in the core $C(N, c)$ of the game $(N, c)$, which is defined as

$$
C(N, c)=\left\{y \in \mathbb{R}^{N} \mid \sum_{i \in N} y_{i}=c(N) ; \sum_{i \in S} y_{i} \leq c(S) \text { for all } S \subset N\right\} .
$$


If the cost is allocated among the players according to an allocation in the core, then any coalition $S$ pays at most its own $\operatorname{costs} c(S)$. Hence, no coalition has an incentive to break up the cooperation with coalition $N$.

We are now ready to view the Jackson network problem as a cooperative cost game.

\subsection{Jackson games}

Based on the optimal capacity allocation of a group of queues in our $n$-node Jackson network, see Subsection 2.1, we define a corresponding cooperative cost game. From now on we refer to this game as a Jackson game.

Definition. A Jackson game is a cost game $(N, c)$ with the set of queues $N=\{1, \ldots, n\}$ as player set. The cost $c(S)$ of coalition $S \subseteq N$ is given by (3).

For each queue in a Jackson game, we define the relative excess capacity value, or simply $r$-value, as the value of the excess capacity, relative to the square root of the arrival rate, i.e.,

$$
r_{i}=\frac{\mu_{i}-\lambda_{i}}{\sqrt{\lambda_{i}}}, \quad i \in N
$$

We also generalize this concept by defining the $r$-value of a coalition $T$ as

$$
\bar{r}_{T}=\frac{\sum_{i \in T}\left(\mu_{i}-\lambda_{i}\right)}{\sum_{i \in T} \sqrt{\lambda_{i}}}, \quad T \subseteq N
$$

Notice that $\bar{r}_{T}$ can be seen as the weighted average of the $r$-values of the queues in $T$, where the weights are the square roots of the arrival rates, i.e.,

$$
\bar{r}_{T}=\sum_{i \in T} \frac{\sqrt{\lambda_{i}}}{\sum_{k \in T} \sqrt{\lambda_{k}}} r_{i} .
$$

Proposition 1. The following properties hold.

(i) Jackson games are not monotone decreasing.

(ii) For all $n \geq 2$, n-node Jackson games may or may not be monotone increasing.

(iii) Jackson games are subadditive.

Proof. For (i), assume without loss of generality that $r_{1} \leq r_{2}$, and let $T=\{1,2\}$ and $S=\{2\}$. Then we have

$$
\bar{r}_{T}=\frac{\sqrt{\lambda_{1}}}{\sqrt{\lambda_{1}}+\sqrt{\lambda_{2}}} r_{1}+\frac{\sqrt{\lambda_{2}}}{\sqrt{\lambda_{1}}+\sqrt{\lambda_{2}}} r_{2} \leq r_{2}
$$

and hence

$$
c(S)=\frac{\sqrt{\lambda_{2}}}{r_{2}}<\frac{\sqrt{\lambda_{1}}+\sqrt{\lambda_{2}}}{r_{2}} \leq \frac{\sqrt{\lambda_{1}}+\sqrt{\lambda_{2}}}{\bar{r}_{T}}=c(T) .
$$

To prove (ii), we construct two concrete examples. A (non-trivial) example of an $n$-node monotone increasing Jackson game can be found by choosing the capacities sufficiently close 
together, e.g. take $\lambda_{i}=1$ and all $\mu_{i}$ inside the interval $[2-\varepsilon, 2+\varepsilon]$ for some $\varepsilon>0$. Then the cost of any $k$-node coalition lies inside $[k /(1+\varepsilon), k /(1-\varepsilon)]$. By taking $\varepsilon<1 /(2 k+1)$ we can ensure that these intervals do not overlap for different $k \leq n$. On the other hand, any $n$-node Jackson game with $\lambda_{1}=\lambda_{2}=\lambda$ and $\mu_{2}>3 \mu_{1}-2 \lambda$ is not monotone increasing since for $T=\{1,2\}$ we have $c(T)=2 \lambda /\left(\bar{\mu}_{T}-\lambda\right)<\lambda /\left(\mu_{1}-\lambda\right)=c(\{1\})$ with $\bar{\mu}_{T}=\sum_{i \in T} \mu_{i} /|T|$ the average capacity of queues in coalition $T$.

To prove (iii) we observe that optimal capacity allocations for coalitions $S$ and $T$ induce a feasible capacity allocation for coalition $S \cup T$ in optimization problem (1).

The fact that Jackson games are usually not monotone is not helpful for the analysis of the core. Before we move on to this in Section 3, we present some weaker monotonicity results. In the following proposition we show that the total cost of any coalition may increase or decrease by adding queues with respectively sufficiently low or high capacity to the coalition.

Proposition 2. Consider two coalitions $S$ and $T$ satisfying $S \subseteq T \subseteq N$. Then $c(S) \leq c(T)$ is equivalent with

$$
\bar{r}_{T \backslash S} \leq\left(1+\frac{\sum_{k \in T} \sqrt{\lambda_{k}}}{\sum_{k \in S} \sqrt{\lambda_{k}}}\right) \bar{r}_{S} .
$$

In the particular case where $T \backslash S$ only contains a single node, say $T=S \cup\{i\}$ with $i \notin S$, we have the following.

(i) A simple sufficient condition for $c(S) \leq c(T)$ (increasing cost when adding queue $i$ to $S$ ) is given by $r_{i} \leq 2 \bar{r}_{S}$.

(ii) When $|S|>2$ a simple sufficient condition for $c(S)>c(T)$ (decreasing cost when adding queue $i$ to $S$ ) is that both $r_{i}>3 \bar{r}_{S}$ and $\sqrt{\lambda_{i}} \leq \sum_{k \in S} \sqrt{\lambda_{k}}$ hold.

Proof. First, we derive

$$
\bar{r}_{T}=\frac{\sum_{k \in S} \sqrt{\lambda_{k}}}{\sum_{k \in T} \sqrt{\lambda_{k}}} \bar{r}_{S}+\frac{\sum_{k \in T \backslash S} \sqrt{\lambda_{k}}}{\sum_{k \in T} \sqrt{\lambda_{k}}} \bar{r}_{T \backslash S} .
$$

Further, the inequality $c(S) \leq c(T)$ is equivalent to

$$
\sum_{k \in S} \sqrt{\lambda_{k}} \bar{r}_{T} \leq \sum_{k \in T} \sqrt{\lambda_{k}} \bar{r}_{S},
$$

or,

$$
\sum_{k \in S} \sqrt{\lambda_{k}}\left(\frac{\sum_{k \in S} \sqrt{\lambda_{k}}}{\sum_{k \in T} \sqrt{\lambda_{k}}} \bar{r}_{S}+\frac{\sum_{k \in T \backslash S} \sqrt{\lambda_{k}}}{\sum_{k \in T} \sqrt{\lambda_{k}}} \bar{r}_{T \backslash S}\right) \leq \sum_{k \in T} \sqrt{\lambda_{k}} \bar{r}_{S} .
$$

Solving for $\bar{r}_{T \backslash S}$ leads to (7). Statements $(i)$ and (ii) follow immediately.

As mentioned before, in the next section we study the core of Jackson games. In particular we want to know if fair cost allocations always exist. That is, if the core is always a nonempty set. 


\section{Main results}

In this section we first derive results on the core of two- and three-node networks. Then we point out how larger networks differ from three-node networks, and present results for these networks.

\subsection{Two-node Jackson games}

For two-node Jackson games, we have the following result.

Theorem 3. Jackson games corresponding to two-node networks have a nonempty core.

Proof. This follows immediately from the subadditivity property (4) of Jackson games, see Remark 1.

We give an example to illustrate that the core can easily be found explicitly in this case.

Example 1. Consider a two-node network with arrival rates $\lambda_{1}=1, \lambda_{2}=4$, and service rates $\mu_{1}=\mu_{2}=5$. The costs of the coalitions are $c(\{1\})=1 / 4, c(\{2\})=4$, and $c(N)=9 / 5$. By Theorem 3 cooperation is worthwhile since cost savings are achieved: $c(N)<c(\{1\})+c(\{2\})$. The core of the game is nonempty and equals

$$
C(N, c)=\{(x, 9 / 5-x) \mid-11 / 5 \leq x \leq 1 / 4\} .
$$

Notice that in this example there exist core allocations such that the cost allocated to the first queue is negative, for example $(-11 / 5,4)$. In such allocations the second queue gets paid for its cooperation. Note that the first queue has the largest excess capacity; its contribution is so valuable that this queue may receive payment to cooperate. This phenomenon is not always present, e.g. when $\mu_{1}=2$, we get $c(\{1\})=1, c(\{2\})=4, c(N)=9 / 2$ and $C(N, c)=$ $\{(x, 9 / 2-x) \mid 1 / 2 \leq x \leq 1\}$.

\section{$3.2 \quad$ Three-node Jackson games}

All the results in this subsection are based on orderings of the $r$-values of the queues. First, we identify two cost allocations that belong to the core.

Theorem 4. Jackson games corresponding to three-node networks have a nonempty core. In particular, if we assume without loss of generality that ${ }^{1}$

$$
r_{1} \geq r_{2} \geq r_{3}
$$

and $\sigma(1)=2$, i.e. queue 2 is in first position, then the marginal vector $m^{\sigma}(c)$ is a cost allocation that belongs to the core of the game.

Proof. There are two marginal vectors in which node 2 is in first position, namely $m^{213}(c)$ and $m^{231}(c)$. Consider the first marginal vector. The marginal contributions of the nodes are

$$
\begin{aligned}
& m_{1}^{213}(c)=c(\{1,2\})-c(\{2\}), \\
& m_{2}^{213}(c)=c(\{2\}), \\
& m_{3}^{213}(c)=c(N)-c(\{1,2\}) .
\end{aligned}
$$

We proceed by checking the core-conditions:

\footnotetext{
${ }^{1}$ If two or three queues have equal $r$-value, either of these can be chosen as 'the' queue with middle $r$-value.
} 
- $m_{1}^{213}(c) \leq c(\{1\})$ : true by subadditivity;

- $m_{2}^{213}(c) \leq c(\{2\})$ : true (with equality);

- $m_{3}^{213}(c) \leq c(\{3\})$ : true by subadditivity;

- $m_{1}^{213}(c)+m_{2}^{213}(c) \leq c(\{1,2\})$ : true (with equality);

- $m_{1}^{213}(c)+m_{3}^{213}(c) \leq c(\{1,3\})$ : true by subadditivity;

- $m_{2}^{213}(c)+m_{3}^{213}(c) \leq c(\{2,3\})$ : see below;

- $m_{1}^{213}(c)+m_{2}^{213}(c)+m_{3}^{213}(c)=c(N)$ : true.

It remains to prove the condition related to coalition $\{2,3\}$, which can be written as

$$
c(\{2\})+c(N)-c(\{1,2\}) \leq c(\{2,3\}),
$$

or as

$$
\left(\frac{\lambda_{2}}{x_{2}}\right)+\left(\frac{\lambda_{1}}{\bar{x}_{1}}+\frac{\lambda_{2}}{\bar{x}_{2}}+\frac{\lambda_{3}}{\bar{x}_{3}}\right) \leq\left(\frac{\lambda_{1}}{\hat{x}_{1}}+\frac{\lambda_{2}}{\hat{x}_{2}}\right)+\left(\frac{\lambda_{2}}{\check{x}_{2}}+\frac{\lambda_{3}}{\check{x}_{3}}\right),
$$

where we define (recall also (2)),

$$
\begin{aligned}
x_{i}=\mu_{i}-\lambda_{i}, & i=1,2,3, \\
\bar{x}_{i}=m_{i,\{1,2,3\}}-\lambda_{i}, & i=1,2,3, \\
\hat{x}_{i}=m_{i,\{1,2\}}-\lambda_{i}, & i=1,2, \\
\check{x}_{i}=m_{i,\{2,3\}}-\lambda_{i}, & i=2,3 .
\end{aligned}
$$

The interpretation of these quantities is that $x_{i}$ is the excess capacity for queue $i$ when considered in isolation (without cooperation), $\bar{x}_{i}$ is the excess capacity for queue $i$ for the optimal capacity allocation in coalition $\{1,2,3\}$, $\hat{x}_{i}$ is the excess capacity for queue $i$ for the optimal capacity allocation in coalition $\{1,2\}$, $\breve{x}_{i}$ is the excess capacity for queue $i$ for the optimal capacity allocation in coalition $\{2,3\}$.

For $\hat{x}_{2}$ we can write, based on (2),

$$
\begin{aligned}
\hat{x}_{2} & =\frac{\sqrt{\lambda_{2}}}{\sqrt{\lambda_{1}}+\sqrt{\lambda_{2}}}\left(\mu_{1}-\lambda_{1}+\mu_{2}-\lambda_{2}\right) \\
& =\frac{\sqrt{\lambda_{2}}}{\sqrt{\lambda_{1}}+\sqrt{\lambda_{2}}}\left(x_{1}+x_{2}\right) \\
& \geq x_{2},
\end{aligned}
$$

where we used $x_{1} \sqrt{\lambda_{2}} \geq x_{2} \sqrt{\lambda_{1}}$, which is due to the ordering in (8). Similarly, we have $\check{x}_{2} \leq x_{2}$. As for $\bar{x}_{1}, \bar{x}_{2}, \bar{x}_{3}$, they satisfy $\bar{x}_{1}+\bar{x}_{2}+\bar{x}_{3}=x_{1}+x_{2}+x_{3}$ and $\bar{x}_{i}+\lambda_{i}, i=1,2,3$, are the optimal solution to (1) for $S=N$. Now we consider a suboptimal solution to this, given by

$$
\tilde{x}_{1}=\hat{x}_{1}, \quad \tilde{x}_{2}=\hat{x}_{2}+\check{x}_{2}-x_{2}, \quad \tilde{x}_{3}=\check{x}_{3} .
$$


Notice that indeed $\tilde{x}_{1}+\tilde{x}_{2}+\tilde{x}_{3}=x_{1}+x_{2}+x_{3}$ as should, because $\hat{x}_{1}+\hat{x}_{2}=x_{1}+x_{2}$ and $\check{x}_{2}+\check{x}_{3}=x_{2}+x_{3}$. Furthermore we have $\tilde{x}_{2}=\hat{x}_{2}-\left(x_{2}-\check{x}_{2}\right) \leq \hat{x}_{2}$ and similarly $\tilde{x}_{2} \geq \check{x}_{2}$. We can now prove (10) as follows,

$$
\begin{aligned}
\left(\frac{\lambda_{2}}{x_{2}}\right) & +\left(\frac{\lambda_{1}}{\bar{x}_{1}}+\frac{\lambda_{2}}{\bar{x}_{2}}+\frac{\lambda_{3}}{\bar{x}_{3}}\right) \\
& \leq \frac{\lambda_{2}}{x_{2}}+\frac{\lambda_{1}}{\tilde{x}_{1}}+\frac{\lambda_{2}}{\tilde{x}_{2}}+\frac{\lambda_{3}}{\tilde{x}_{3}} \\
& =\frac{\lambda_{2}}{x_{2}}+\frac{\lambda_{1}}{\hat{x}_{1}}+\frac{\lambda_{2}}{\tilde{x}_{2}}+\frac{\lambda_{3}}{\check{x}_{3}} \\
& \leq \frac{\lambda_{2}}{\hat{x}_{2}}+\frac{\lambda_{1}}{\hat{x}_{1}}+\frac{\lambda_{2}}{\check{x}_{2}}+\frac{\lambda_{3}}{\check{x}_{3}},
\end{aligned}
$$

where the first inequality is due to the optimality of $\bar{x}_{i}+\lambda_{i}, i=1,2,3$, in (1) for $S=N$, and the second inequality is due to the convexity of the function $\lambda_{2} / x$ in $x$ and the fact that both $x_{2}$ and $\tilde{x}_{2}$ lie in the interval $\left[\check{x}_{2}, \hat{x}_{2}\right]$.

For the second marginal vector $m^{231}(c)$, the marginal contributions of the nodes are

$$
\begin{aligned}
& m_{1}^{213}(c)=c(N)-c(\{2,3\}), \\
& m_{2}^{213}(c)=c(\{2\}), \\
& m_{3}^{213}(c)=c(\{2,3\})-c(\{2\}) .
\end{aligned}
$$

As above, the core-conditions can be checked to hold, either with equality, or by subadditivity. The only exception is the condition related to coalition $\{1,2\}: c(N)-c(\{2,3\})+c(\{2\}) \leq$ $c(\{1,2\})$, which is equivalent to (9) and therefore also holds. This proves the result.

The theorem above shows that also three-node Jackson games have a nonempty core. Notice that any convex combination of the two above-mentioned marginal vectors also belongs to the core because this is a convex set.

Cost allocations in the core of three-node Jackson games may have negative elements - the corresponding queue receives payment to cooperate - as was the case for two-node networks. For example, consider the situation in Example 1, and add a third queue with demand rate $\lambda_{3}=4$ and capacity $\mu_{3}=5$. According to Theorem 4 , the cost allocation $m^{321}(c)=(-23 / 6,4,4)$ belongs to the core of the game. Node 1 has the largest excess service capacity, and may be paid for cooperation.

The two particular cost allocations identified in Theorem 4 are such that the queue with middle $r$-value does not gain from cooperation. That is, the cost that is allocated to this queue is the same as its stand-alone cost $c(\{i\})$. Below we characterize the conditions under which all cost allocations in the core have this property.

Proposition 5. Consider a three-node Jackson network. If $r_{2}=\bar{r}_{\{1,3\}}$ then

1. $c(\{2\})+c(\{1,3\})=c(N)$,

2. $c(\{2\}) / c(N)=\sqrt{\lambda_{2}} /\left(\sqrt{\lambda_{1}}+\sqrt{\lambda_{2}}+\sqrt{\lambda_{3}}\right)$,

3. node 2 has no strict gain from cooperation, and

4. the core $C(N, c)$ is the convex hull of the two marginal vectors $m^{213}(c)$ and $m^{231}(c)$. 


\begin{tabular}{|r|cccc|cccccc|}
\hline$S$ & $\{1\}$ & $\{2\}$ & $\{3\}$ & $\{4\}$ & $\{1,2\}$ & $\{1,3\}$ & $\{1,4\}$ & $\{2,3\}$ & $\{2,4\}$ & $\{3,4\}$ \\
$c(S)$ & $1 / 7$ & $1 / 5$ & $1 / 3$ & 1 & $1 / 3$ & $2 / 5$ & $1 / 2$ & $1 / 2$ & $2 / 3$ & 1 \\
\hline$S$ & $\{1,2,3\}$ & $\{1,2,4\}$ & $\{1,3,4\}$ & $\{2,3,4\}$ & $\{1,2,3,4\}$ & & & & & \\
$c(S)$ & $3 / 5$ & $9 / 13$ & $9 / 11$ & 1 & 1 & & & & & \\
\hline
\end{tabular}

Table 1: Costs of all coalitions for the game in Example 2.

Proof. Assuming $r_{2}=\bar{r}_{\{1,3\}}$, we write for the first statement

$$
c(\{2\})+c(\{1,3\})=\frac{\sqrt{\lambda_{2}}}{r_{2}}+\frac{\sqrt{\lambda_{1}}+\sqrt{\lambda_{3}}}{\bar{r}_{\{1,3\}}}=\frac{\sqrt{\lambda_{1}}+\sqrt{\lambda_{2}}+\sqrt{\lambda_{3}}}{\bar{r}_{\{1,2,3\}}}=c(N),
$$

where the second equality follows from

$$
\bar{r}_{\{1,2,3\}}=\frac{\sqrt{\lambda_{2}}}{\sum_{k \in N} \sqrt{\lambda_{k}}} r_{2}+\frac{\sqrt{\lambda_{1}}+\sqrt{\lambda_{3}}}{\sum_{k \in N} \sqrt{\lambda_{k}}} \bar{r}_{\{1,3\}}=r_{2}=\bar{r}_{\{1,3\}} .
$$

The second statement follows from

$$
\frac{c(\{2\})}{c(N)}=\frac{\sqrt{\lambda_{2}} / r_{2}}{\left(\sqrt{\lambda_{1}}+\sqrt{\lambda_{2}}+\sqrt{\lambda_{3}}\right) / \bar{r}_{\{1,2,3\}}},
$$

again combined with $r_{2}=\bar{r}_{\{1,2,3\}}$.

For the third statement, first note that any allocation $x$ in the core should satisfy $x_{2} \leq$ $c(\{2\})$ and $x_{1}+x_{3} \leq c(\{1,3\})$. Together with the efficiency condition $x_{1}+x_{2}+x_{3}=c(N)$ this leads to $x_{2}=c(\{2\})$ for any allocation in the core. Hence, node 2 does not gain from cooperation. Together with Theorem 4 this implies that any element of the core $C(N, c)$ is a convex combination of the two marginal vectors $m^{213}(c)$ and $m^{231}(c)$.

Remark 2. One may be inclined to argue that when a player does not gain from cooperation, as in the context of Proposition 5, he may refrain from cooperation to prevent extra benefit for the other players. However, it is easy to see (from the first statement) that his decision to cooperate or not does not affect the cost paid, or amount gained, by the other players.

\subsection{Jackson games with more than three nodes}

For three-node Jackson networks we showed that the core of the corresponding game is nonempty by identifying two marginal vectors that belong to the core, see Theorem 4 . This approach cannot be extended to four-node networks, as the following example illustrates.

Example 2. Consider the four-node tandem Jackson network with arrival rates $\lambda_{i}=1$, $i=1, \ldots, 4$, and service rates $\mu_{1}=8, \mu_{2}=6, \mu_{3}=4$ and $\mu_{4}=2$. The costs of the various coalitions in the corresponding Jackson game are given in Table 1. The 24 marginal vectors are listed in Table 2. One can verify that none of these marginal vectors belongs to the core of the game. However, the core of the game is nonempty since, for example, the allocation $x=(15,180,315,480) / 990$ belongs to the core. 


\begin{tabular}{|llllll|llllll|}
\hline$m^{1234}$ & $m^{1243}$ & $m^{1324}$ & $m^{1342}$ & $m^{1423}$ & $m^{1432}$ & $m^{2134}$ & $m^{2143}$ & $m^{2314}$ & $m^{2341}$ & $m^{2413}$ & $m^{2431}$ \\
\hline 0.143 & 0.143 & 0.143 & 0.143 & 0.143 & 0.143 & 0.133 & 0.133 & 0.100 & 0 & 0.026 & 0 \\
0.190 & 0.190 & 0.400 & 0.182 & 0.192 & 0.182 & 0.200 & 0.200 & 0.200 & 0.200 & 0.200 & 0.200 \\
0.267 & 0.308 & 0.257 & 0.257 & 0.308 & 0.318 & 0.267 & 0.308 & 0.300 & 0.300 & 0.308 & 0.333 \\
0.400 & 0.359 & 0.200 & 0.418 & 0.357 & 0.357 & 0.400 & 0.359 & 0.400 & 0.500 & 0.467 & 0.467 \\
\hline
\end{tabular}

\begin{tabular}{|llllll|llllll|}
\hline$m^{3124}$ & $m^{3142}$ & $m^{3214}$ & $m^{3241}$ & $m^{3412}$ & $m^{3421}$ & $m^{4123}$ & $m^{4132}$ & $m^{4213}$ & $m^{4231}$ & $m^{4312}$ & $m^{4321}$ \\
\hline 0.067 & 0.067 & 0.100 & 0 & -0.182 & 0 & -0.500 & -0.500 & 0.026 & 0 & -0.182 & 0 \\
0.200 & 0.182 & 0.167 & 0.167 & 0.182 & 0 & 0.192 & 0.182 & -0.333 & -0.333 & 0.182 & 0 \\
0.333 & 0.333 & 0.333 & 0.333 & 0.333 & 0.333 & 0.308 & 0.318 & 0.308 & 0.333 & 0 & 0 \\
0.400 & 0.418 & 0.400 & 0.500 & 0.667 & 0.667 & 1 & 1 & 1 & 1 & 1 & 1 \\
\hline
\end{tabular}

Table 2: The 24 marginal vectors of the game in Example 2. Top to bottom entries correspond to players 1 till 4 .

We need a different approach to study the core of the four-node Jackson game. First we show some simple structural results. If two queues $i$ and $j$ have equal $r$-values, then their joint cost $c(\{i, j\})$ is the sum of their individual costs. This follows from the more general statement below by taking $S=\{i\}$ and $T=\{j\}$ :

Proposition 6. Consider an n-node Jackson network with $\bar{r}_{S}=\bar{r}_{T}$ for some disjoint coalitions $S, T \subset N, S \cap T=\emptyset$. Then $c(S \cup T)=c(S)+c(T)$ in the corresponding Jackson game.

Proof. First note that $\bar{r}_{S \cup T}=\bar{r}_{S}=\bar{r}_{T}$. Then

$$
c(S \cup T)=\frac{\sum_{i \in S \cup T} \sqrt{\lambda_{i}}}{\bar{r}_{S \cup T}}=\frac{\sum_{i \in S} \sqrt{\lambda_{i}}}{\bar{r}_{S}}+\frac{\sum_{i \in T} \sqrt{\lambda_{i}}}{\bar{r}_{T}}=c(S)+c(T) .
$$

Next, if all queues have equal $r$-values, then there is a unique core allocation in which each queue pays its own cost as if in isolation, proportional to the square root of the arrival rate.

Proposition 7. Consider an n-node Jackson network in which all nodes have equal $r$-value, $r$ say. Then the core of the Jackson game consists of a single allocation $x$ with $x_{i}=\lambda_{i} /\left(\mu_{i}-\right.$ $\left.\lambda_{i}\right)=\sqrt{\lambda_{i}} / r$ for all $i$.

Proof. If $r_{i}=r$ for all $i \in N$, then also $r_{S}=r$ for any coalition $S$. Therefore the cost of coalition $S$ equals

$$
c(S)=\frac{\sum_{i \in S} \sqrt{\lambda_{i}}}{\bar{r}_{S}}=\sum_{i \in S} \frac{\sqrt{\lambda_{i}}}{r_{i}}=\sum_{i \in S} \frac{\lambda_{i}}{\mu_{i}-\lambda_{i}}=\sum_{i \in S} c(\{i\}) .
$$

The game is additive, so there is a unique core-allocation $x$ with $x_{i}=\lambda_{i} /\left(\mu_{i}-\lambda_{i}\right)$ for all $i$. 
We now come to our final result. Assuming that for certain parameter settings the core would be empty, we tried to numerically find such parameters. However, we always found a small subset of allocations that satisfy all core conditions. By careful analysis we were then able to find an explicit expression for a particular cost allocation that always seemed to be in the core. In the theorem below we specify this allocation for general Jackson games, and prove that indeed it always belongs to the core.

Theorem 8. Consider an n-node Jackson network. The cost allocation $x$ with

$$
x_{i}=\left(2 \frac{\sqrt{\lambda_{i}}}{\sum_{j \in N} \sqrt{\lambda_{j}}}-\frac{\mu_{i}-\lambda_{i}}{\sum_{j \in N}\left(\mu_{j}-\lambda_{j}\right)}\right) c(N)
$$

belongs to the core of the corresponding Jackson game. For any coalition $S$ the cost under this allocation is in fact strictly less than $c(S)$, unless $\bar{r}_{S}=\bar{r}_{N}$.

Proof. It follows from the definition of $x$ that this allocation is efficient, i.e. $\sum_{i \in N} x_{i}=c(N)$. We continue by noting that the core condition for any coalition $S$, i.e., $\sum_{i \in S} x_{i} \leq c(S)$, is equivalent to

$$
2 \leq \frac{\sum_{j \in N} \sqrt{\lambda_{j}}}{\sum_{j \in S} \sqrt{\lambda_{j}}}\left(\frac{c(S)}{c(N)}+\frac{\sum_{j \in S}\left(\mu_{j}-\lambda_{j}\right)}{\sum_{j \in N}\left(\mu_{j}-\lambda_{j}\right)}\right) .
$$

Since this can be rewritten as

$$
2 \leq \frac{\bar{r}_{N}}{\bar{r}_{S}}+\frac{\bar{r}_{S}}{\bar{r}_{N}}
$$

and since $a+a^{-1} \geq 2$ for any $a>0$ (with equality holding only for $a=1$ ), both statements of the theorem follow.

We note that the cost allocation in Theorem 8 may also lead to negative cost in case of widely varying $r$-values. For instance, in the setting of Example 1, the resulting cost allocation is $x=(-18 / 75,153 / 75)$, which has a negative cost component for queue 1 .

Interestingly, the cost allocation in Theorem 8 can also be given an interpretation by rewriting it as

$$
x_{i}=\frac{\lambda_{i}}{m_{i, N}-\lambda_{i}}-\alpha_{N}\left(m_{i, N}-\mu_{i}\right)
$$

where

$$
\alpha_{N}=-\frac{\left.\left(\sum_{k} \sqrt{\lambda_{k}}\right)\right)^{2}}{\left(\sum_{k}\left(\mu_{k}-\lambda_{k}\right)^{2}\right.}=-1 / \bar{r}_{N}^{2}
$$

is the Lagrange multiplier for the minimization problem in (1), for the grand coalition $S=N$. Thus, in addition to their 'own cost' after the capacity reallocation, all players pay (or receive) an additional amount which is linear in the capacity increase (or decrease) they experience, weighted with the (negative) shadow price of one unit of capacity. In fact, the form (11) can also be found from [11], where it is also shown that for so-called "market games", a nonempty core is equivalent to the game being balanced. Indeed our game is such a balanced market game.

Based on Theorem 8, or on the above, we can conclude

Corollary 9. Jackson games always have a nonempty core.

Hence, a stable basis for cooperation always exists. 


\section{Special case and extension}

\subsection{Tandem games}

When all arrival rates are equal, $\lambda_{i} \equiv \lambda$, say, as is the case in a tandem queue network (see also [12]), some of the results take on a simpler form. The role of the $r$-values as criterion value for many of the results is now simply played by the excess capacities $\mu_{i}-\lambda$, or indeed by the capacities $\mu_{i}$. For convenience sake we highlight the main results for this particular setting.

Let $|S|$ be the number of queues in coalition $S$, and $\bar{\mu}_{S}$ the mean capacity of the queues in $S$. Then first of all, the optimal capacity allocation for coalition $S$ is simply to share the total capacity equally between all queues in $S$, so (2) becomes $m_{i, S}=\bar{\mu}_{S}=\sum_{i \in S} \mu_{i} /|S|$, with total cost $c(S)=|S| \lambda /\left(\bar{\mu}_{S}-\lambda\right)$. Next, the monotonicity condition (7) in Proposition 2 simplifies to

$$
\bar{\mu}_{T \backslash S} \leq \bar{\mu}_{S}+\frac{|T|}{|S|}\left(\bar{\mu}_{S}-\lambda\right) .
$$

Theorems 3 and 4, and Propositions 5, 6 and 7 continue to hold under the same respective assumptions, in which $r$-values can always be simply replaced by the corresponding $\mu$-values (the second statement of Proposition 5 then becomes $c(\{2\}) / c(N)=1 / 3$ ). Finally, the particular cost allocation in Theorem 8 , simplifies to

$$
x_{i}=\left(2-\frac{\mu_{i}-\lambda}{\bar{\mu}_{N}-\lambda}\right) \frac{c(N)}{|N|}
$$

This belongs to the core of the game, and for any coalition $S$ the cost under this allocation is strictly less than $c(S)$, unless $\bar{\mu}_{S}=\bar{\mu}_{N}$.

\subsection{Utilization as cost}

We now extend our results to a different cost structure, namely to the case in which the cost of queue $i$ is given by the server utilization, instead of the expected number in queue. This may be useful when the operator is more interested in the direct cost of operation, rather than the delay performance for the customers. Thus, we take $c(i)=\lambda_{i} / \mu_{i}$ and replace the minimization problem for coalition $S$ in (1) by

$$
\begin{aligned}
\min _{m_{i}, i \in S} & \sum_{i \in S} \frac{\lambda_{i}}{m_{i}} \\
\text { s.t. } & \sum_{i \in S} m_{i}=\sum_{i \in S} \mu_{i}, \quad m_{i} \geq \lambda_{i} .
\end{aligned}
$$

The second set of constraints $m_{i} \geq \lambda_{i}$ is included to ensure that the utilizations never exceed 1 . In fact for stability of the queue sizes we need these inequalities to be strict, but then a solution may not exist.

Since solving the solution to this problem is cumbersome, we restrict ourselves to the assumption that all arrival rates are equal $\left(\lambda_{i}=\lambda\right.$, say), as in the previous subsection. The optimal capacity allocation is then to share the total capacity equally between all queues in $S$, i.e. $m_{i, S}=\bar{\mu}_{S}=\sum_{i \in S} \mu_{i} /|S|$, where $|S|$ is the number of queues in coalition $S$. Notice 
that indeed $\mu_{S}>\lambda$ if for all $i \in N$ we have $\mu_{i}>\lambda$, as we assume. The total cost for coalition $S$ is obviously given by $c(S)=|S| \lambda / \bar{\mu}_{S}$.

In this setting it is possible to find a similar cost allocation that lies in the core as in Theorem 8 , or rather as in (12). It is given by

$$
x_{i}=\left(2-\frac{\mu_{i}}{\bar{\mu}_{N}}\right) \frac{c(N)}{|N|} .
$$

This belongs to the core of the game, and for any coalition $S$ the cost under this allocation is strictly less than $c(S)$, unless $\bar{\mu}_{S}=\bar{\mu}_{N}$.

\section{Conclusions and future work}

\subsection{Conclusions}

We considered a Jackson network of queues in which each queue has an independent operator, and investigated whether or not these operators are willing to cooperate in order to reduce the total waiting cost, which is linear in the expected queue lengths (or equivalently, in the expected waiting times). Such a cooperation will involve sharing the individual service capacities, and then dividing the resulting total cost in some way. Our main conclusions are as follows:

- The core of the corresponding cooperative game is never empty. That is, there always exists a cost allocation such that the (operators of the) individual queues have an incentive to cooperate.

- One specific cost allocation has been found explicitly, see Theorem 8. This allocation is strictly beneficial for each coalition, unless the so-called relative excess capacity value of a coalition equals that of the grand coalition.

- The so-called relative excess capacity value, or simply r-value, as just mentioned, turns out to be an important quantity throughout the analysis. It is defined as the (total) excess capacity of a (coalition of) queue(s), divided by (the sum of) the square root(s) of the arrival rate(s), see (5) and (6).

- For the case with two queues, the core can be characterized explicitly as the convex combination of the two marginal vector allocations; for the case with three queues, the core always contains two specific marginal vectors; for cases with more queues the core may contain no marginal vectors at all.

- In case of large asymmetries between queues in terms of arrival and/or service rates (more precisely, in case of large differences in $r$-value), core allocations can be such that some queue(s) have negative cost. In other words, such queues may receive payment from the other queues for the cooperation.

- Similar results are found when the cost is not based on expected waiting times or queue lengths, but on server utilizations. 


\subsection{Future work}

As mentioned in the introduction, many assumptions can be done within our framework of 'queueing network games'. In particular we intend to study another way of "sharing capacity", in which the routing pattern is changed such that 'underloaded' queues can provide service to jobs that otherwise would have been routed to other (highly loaded) queues. Also, we will consider dynamic ways of cooperation, and stochastic cost structures.

\section{References}

[1] E. Altman, T. Boulogne, R. El-Azouzi, T. Jiménez, and L. Wynter. A survey on networking games in telecommunications. Computers \& Operations Research, 33(2):286 311, 2006.

[2] S. Andradóttir and H. Ayhan. Throughput maximization for tandem lines with two stations and flexible servers. Operations Research, 53:516-531, 2005.

[3] S. Anily and M. Haviv. Cooperation in Service Systems. Operations Research, 58(3):660673, 2010.

[4] M.D. García-Sanz, F.R. Fernández, M.G. Fiestras-Janeiro, I. García-Jurado, and J. Puerto. Cooperation in Markovian queueing models. European J. Oper. Res., 188(2):485-495, 2008.

[5] R. Gibbens and P. Key. Coalition games and resource allocation in ad-hoc networks. In Pietro Li, Eiko Yoneki, Jon Crowcroft, and Dinesh Verma, editors, Bio-Inspired Computing and Communication, volume 5151 of Lecture Notes in Computer Science, pages 387-398. Springer Berlin / Heidelberg, 2008.

[6] R.J. Gibbens, F.P. Kelly, G.A. Cope, and M.J. Whitehead. Coalitions in the international network. In A. Jensen and V.B. Iversen, editors, 13th International Teletraffic Congress (ITC), 1991, pages 93-91, 1991.

[7] P. González and C. Herrero. Optimal sharing of surgical costs in the presence of queues. Math. Methods Oper. Res., 59(3):435-446, 2004.

[8] L. Kleinrock. Communication nets: stochastic message flow and delay. McGraw-Hill, New York, 1964.

[9] A. Mandelbaum and M.J. Reiman. On pooling in queueing networks. Management Science, 44:971-981, 1998.

[10] H. Peters. Game theory. Springer-Verlag, Berlin, 2008.

[11] L.S. Shapley and M. Shubik. Market games. Journal of Economic Theory, 1(1):9-25, 1969.

[12] J. Timmer and W. Scheinhardt. How to share the cost of cooperating queues in a tandem network? In Teletraffic Congress (ITC), 2010 22nd International, pages 1 -7, 2010.

[13] J. Walrand. An introduction to queueing networks. Prentice-Hall, New Jersey, 1988. 